Published in final edited form as:

ECS Trans. 2014 ; 64(4): 499-505. doi:10.1149/06404.0499ecst.

\title{
Cellulose-Chitosan-Keratin Composite Materials: Synthesis, Immunological and Antibacterial Properties
}

\author{
Meghann Rosewald ${ }^{a}$, Fang Yao Stephen Hou ${ }^{a}$, Tamutsiwa Mututuvari ${ }^{b}$, April L. Harkins ${ }^{a}$, \\ and Chieu D. Tran ${ }^{b}$ \\ a Dept of Clinical Laboratory Science, Marquette University, Milwaukee, WI 53201 \\ ${ }^{b}$ Department of Chemistry, Marquette University, Milwaukee, WI 53201
}

\begin{abstract}
Novel composites were synthesized from keratin (KER), cellulose (CEL) and chitosan (CS). The method is recyclable because majority $(>88 \%)$ of $\left[\mathrm{BMIm}^{+} \mathrm{Cl}^{-}\right]$, an ionic liquid (IL), used as the sole solvent, was recovered for reuse. Experimentally, it was confirmed that unique properties of each component remain intact in the composites, namely bactericide (from KER and CS) and antiinflammatory property (from KER). Specifically, the composites were examined for their antiinflammatory influence on macrophages. The cells were imaged and immunophenotyped to determine the quantity using the macrophage marker CD11b. The 75:25 [KER+CS] composite was found to have the least amount of CD11b macrophages compared to other composites. Bactericidal assays indicated that all composites, except the 25:75 [KER+CS], substantially reduce the growth of organisms such as vancomycin resistant Enterococcus (VRE) and Eschericia coli. The results clearly indicate that the composites possess all properties needed for effective use as a wound dressing.
\end{abstract}

\section{Introduction}

Non-antigenic keratin is known to possess advantages for wound care, tissue reconstruction, cell seeding and diffusion, and drug delivery as topical or implantable biomaterial [1-5]. As implantable film, sheet, or scaffold, keratin can be absorbed by surrounding tissue to provide structural integrity within the body while maintaining stability under mechanical load, and in time can break down to leave neo-tissue. Keratin is found to be characteristically abundant in cysteine residues (7-20\% of the total amino acid residues) [1-5]. These cysteine residues are oxidized to give inter- and intra-molecular disulfide bond, which results in threedimensionally linked network of keratin fiber. Interestingly, in spite of its unique structure, keratin has relatively poor mechanical properties, and as a consequence, it was not possible to fully exploit unique properties of keratin for various applications [1-5].

Polysaccharides such as cellulose (CEL) are known to have strong mechanical property [6-8]. Similar to CEL, chitosan (CS), another polysaccharide derived from chitin, also has strong mechanical property in addition to its ability to stop bleeding (hemostasis), heal wounds, kill bacteria and adsorb organic and inorganic pollutants [6-8].

The information presented is indeed provocative and clearly indicate that adding polysaccharide such as CEL and/or CS to KER would substantially enhance the mechanical 
property of the composite to enable it to be used for various applications. Such consideration prompted us to initiate this study which aim to improve the mechanical properties of the KER composites by adding either CEL or CS to the composites, and to demonstrate bactericide and the anti-inflammatory properties of the [KER+CEL/CS] composites by quantitating and comparing the relative mean fluorescent intensity of macrophage activation using flow cytometry method. Results of our initial investigation are reported herein.

\section{Materials and Methods}

\section{Materials}

Chitosan $(M W \approx 310-375 \mathrm{kDa})$, derived from chitin from crustacean shells and microcrystalline cellulose $(\mathrm{DP} \approx 300)$ [3], derived from cotton linters, were purchased from Sigma-Aldrich (Milwaukee, WI). The degree of deacetylation of chitosan, determined by FT-IR, was found to be $84 \pm 2 \%$ [5-7]. Raw sheep (untreated) wool, obtained from a local farm, was cleaned by Soxhlet extraction using a 1:1 (v/v) acetone/ethanol mixture at $80 \pm 3$ ${ }^{\circ} \mathrm{C}$ for $48 \mathrm{~h}$. The wool was then rinsed with distilled water and dried at $100 \pm 1{ }^{\circ} \mathrm{C}$ for $12 \mathrm{~h}$ [9]. 1-Methylimidazole and n-chlorobutane (both from Alfa Aesar, Ward Hill, MA) were distilled and subsequently used to synthesize $\left[\mathrm{BMIm}^{+} \mathrm{CL}^{-}\right][1]$.

The preparation and complete characterization of the $[\mathrm{KER}+\mathrm{CEL} / \mathrm{CS}]$ composite materials used in this study was described in our publications [6-8]. Minimal essential medium (MEM), 10\% Fetal Bovine Serum (FBS) and gentamicin were obtained from Atlanta Biologicals, Inc., Lawerenceville, GA. CellTiter 96® Aqueous Non-Radioactive Cell Proliferation Assay was obtained from Promega, Madison, WI. 1\% Penicillin Streptomycin and phorbol 12-myristate 13-acetate (PMA) were obtained from VWR, Radnor, PA. The enzyme-linked immunosorbent assay (ELISA) kit was from R\&D Systems, Minneapolis, $\mathrm{MN}$ and was used according to the manufacturer's instructions. Images of the fibroblasts and macrophages were taken with an Olympus microscopic camera using CellSens Imaging Software (Olympus, Center Valley, PA). Kendall - Curity AMD gauze sponges, 8 and 12 ply (Covidien, Mansfield, MA), Promogran Matrix wound dressing (Johnson \& Johnson, New Brunswick, NJ), Aquacel and DuoDERM CGF (ConvaTec, Princeton, NJ), Allevyn adhesive (Smith + Nephew, Andover, MA) are wound dressing material that was purchased commercially.

\section{Bactericidal Activity}

Bacterial killing assays were performed in the presence and absence of the composites with different compositions and concentrations. The bacterial strains used in this protocol included Escherichia coli (ATCC 8739), Staphylococcus aureus (ATCC 25923), methicillin resistant S. aureus (ATCC 33591) and vancomycin resistant Enterococcus faecalis (ATCC 51299). The strains were maintained on blood agar at $4^{\circ} \mathrm{C}$. According to a modified protocol from Pinto et. al [10] bacterial cells were grown in nutrient broth for $18-20 \mathrm{hr}$ at $37^{\circ} \mathrm{C}$ with agitation. The cells were diluted in fresh medium and incubated for $24 \mathrm{hr}$ at $37^{\circ} \mathrm{C}$ in the presence of the composites. Serial dilutions of the bacteria were plated onto nutrient agar and incubated for $24 \mathrm{hr}$. Bacterial colony forming units (CFUs) were quantified and compared to bacteria grown in the absence of composites. 


\section{Cell Culture}

The human monocytic cell line THP-1 (ATCC TIB-202) was cultured in RPMI-1640 medium supplemented with $10 \%$ FBS and 1\% Penicillin-Streptomycin. Stimulation and differentiation of monocytes to macrophages was performed by adding $0.2 \mu \mathrm{M}$ phorbol 12 myristate 13-acetate (PMA) to the medium. The cell line was maintained under 5\% CO2 humidified atmospheric conditions in a $37^{\circ} \mathrm{C}$ incubator. Composite materials with different compositions and concentrations (100\% CEL, 75:25 [CEL+KER], 25:75 [CEL+KER], 100\% CS, 75:25 [CS+KER], 25:75 [CS+KER], and 100\% KER) were incubated with THP-1 cells for three days in the presence or absence of $0.2 \mathrm{mM}$ phorbol 12-myristate 13acetate (PMA). Cells without PMA and cells without composite material served as the negative controls. THP-1 cells, after 3 days of incubation in various combinations, were harvested by scrapping. Cells from each condition were diluted in the staining buffer, containing $0.5 \%$ bovine serum albumin (Sigma A2153, St. Louis, MO) to reduce nonspecific binding. The final volume was $1 \mathrm{~mL}$ in each condition. Antibody staining was carried out by adding 1L CD11b PE (Beckman Coulter Immunotech IM 2581) into each cell suspension for 20 minutes at room temperature. Without a wash step, all tubes were ready for acquisition on a Beckman Coulter Cytomics flow cytometer FC 500. Data analysis was performed using the CXP flow cytometry software. We defined cellular debris based on FSC/SSC dotplot for each condition. The expression of CD11b was then displayed on a histogram excluding cellular debris. Mean fluorescence intensity (MFI) from CD11b positive populations was used to assess the degree of activation in each condition.

\section{Results and Discussion}

\section{Mechanical Properties}

As described above, in spite of its potentials, applications of KER have not been fully realized because it has relatively poor mechanical properties [1-5]. Since polysaccharides such as CEL and CS are known to have remarkable high mechanical strength [6-8], it may be possible to improve the mechanical property of KER by adding either CEL or CS to KER to form composites. Accordingly, experiments were systematically designed to verify effect of addition either CEL or CS on the tensile strength of the resultant [KER+CEL/CS] composites. Figure 1 plots tensile strength of $[\mathrm{KER}+\mathrm{CEL}]$ and $[\mathrm{KER}+\mathrm{CS}]$ composite as a function of CEL and CS content, respectively. As illustrated, the tensile strength of [CEL + KER] composite films was found to increase concomitantly with the content of CEL. For example, the tensile strength of [KER $+\mathrm{CEL}]$ increased by at least $4 \mathrm{X}$ when CEL loading was increased from $25 \%$ to $75 \%$. It is worth noting that [KER+CEL] composites are relatively weaker than [CS+CEL] composites which we synthesized previously [6]. For example, $[\mathrm{CEL}+\mathrm{KER}]$ and $[\mathrm{CEL}+\mathrm{CS}]$ containing $75 \%$ and $71 \% \mathrm{CEL}$ had tensile strengths $36 \pm 3 \mathrm{MPa}$ and $52 \mathrm{MPa}$ respectively. This could be attributed to the fact that CEL structure is more similar to that of CS than to KER structure. Therefore relatively stronger interactions may be possible between CEL and CS than between CEL and KER. Although CS also leads to an increase in the tensile strength of $[\mathrm{KER}+\mathrm{CS}]$, its effect is noticeably weaker than that of CEL of comparable loading. For example, [CEL+KER] and [CS+KER] had tensile strength values of $37 \pm 6 \mathrm{MPa}$ and $20 \pm 1 \mathrm{MPa}$ respectively for a $40 \% \mathrm{KER}$ loading. This is hardly surprising considering the fact that CS is inherently weaker than CEL as can be seen 
by the tensile strengths of $100 \% \mathrm{CS}(36 \pm 9 \mathrm{MPa})$ and $100 \% \mathrm{CEL}(82 \pm 4 \mathrm{MPa})$. It is noteworthy to add that $100 \%$ KER was too brittle to measure the tensile strength.

\section{Anti-inflammatory Properties}

CD11b expression is known to be one of the activation markers for monocytes [11]. Furthermore, density of the CD11b molecules on cell surface can be determined by measuring MFI as MFI is known to be a reliable indicator for the density of the CD11b. Accordingly, by using digital imaging and immunophenotyped macrophages to quantitate CD11 through MFI, it was possible for us to determine the anti-inflammatory effect of the composite materials. As expected, it was found that MFI increased significantly following PMA treatment in THP-1 cell line. As MFI is a reliable indicator for the density of CD11b molecules on cell surface, higher MFI values indicating more CD11b molecules on cell surface, or more inflammation. The histograms displaying CD11b fluorescence from mitogen stimulated monocytes is shown in Figure 2. As shown in the figure, the presence of KER and/or CS decreased CD11b values while CEL maintained the same CD11b value as cells treated with PMA. Among all composites tested, the least amount of CD11b macrophages was found in the presence of the 75:25 [CS+KER] composite whereas the $100 \%$ CEL composite brought the highest amount of CD11b macrophages. The results seems to indicate that KER and/or CS can effectively serve as anti-inflammatory agents.

\section{Antibacterial Properties}

We have previously shown that $[\mathrm{CEL}+\mathrm{CS}]$ composites can effectively inhibit the growth of both Gram positive and negative microorganisms [6-8]. For examples, the 100\% chitosan material was found to reduce growth of Escherichia coli (ATCC 8739) and vancomycin resistant Enterococcus faecalis (ATCC 51299) (VRE) by 78\% and 64\%, respectively [8]. Since KER also known to possess antimicrobial activity [1-5,12,13], it is important to determine if KER retains its bactericide upon regenerated and formed composites chitosan. Accordingly, antimicrobial assay of [CEL+KER] and [CS+KER] composites were performed and the results obtained are shown in Figure 3

To assess the antimicrobial activity of the $[\mathrm{KER}+\mathrm{CEL} / \mathrm{CS}]$ composites, bacteria were grown in the presence of the composites for 24 hours. The cultures were then plated onto nutrient rich media and colony forming units were counted and compared to the standard growth control. The results for the percent growth reduction of VRE and E. coli in the presence of the composites are presented in Figure 3. As illustrated, the composites containing CS had the greatest effect on reducing the growth of VRE. Moreover, it should be noted that all of the composites reduced the growth of VRE. The most effective antimicrobial activity against E. coli included those composites with 100\% KER, 100\% CS and 75:25 [KER+CS]. It is noted that the reduction in E. coli correlated with the amount of CS in the composite, the more CS present, the greater is the reduction. While the 100\% KER composite also had a great effect on E. coli growth, although some of the activity was lost when combined with CEL or CS, especially the 25:75 [KER+CS] composite. Experiments are currently under progress to investigate this discrepancy. 


\section{Conclusions}

In summary, we have successfully synthesized novel, biocompatible and biodegradable composite materials from CEL, CS and KER. We have experimentally confirmed that unique properties of each component remain intact in the composites. Specifically, results of tensile strength measurements clearly indicate that adding CEL or CS into KER substantially increases tensile strength of the composites. The tensile strength of [KER $+\mathrm{CEL} / \mathrm{CS}$ ] composites was found to increase concomitantly with the content of CEL or CS. For example, the tensile strength of [KER+ CEL] increased by at least $4 \mathrm{X}$ when CEL loading was increased from $25 \%$ to $75 \%$. The tensile strength of the composite material can be adjusted to any value by adding judicious amount of either CEL or CS. Antiinflammatory effect of the composites, determined by cytometry through immunophenotyped macrophages to quantitate CD11 indicate that the presence of KER and/or CS enable the composite effectively serve as an anti-inflammatory agents. Results of in vitro antibacterial assays confirm that unique properties of CS and KER remain intact in the material, namely, the composite material retain its antibacterial property. The [KER $+\mathrm{CEL} / \mathrm{CS}]$ composites can effectively inhibit growth of VRE and E. coli. Taken together, the results obtained clearly indicate that the composites possess all properties needed for effective use as a wound dressing.

\section{Acknowledgment}

The authors are grateful to Ms. Anna J Frazier for her competent technical assistance. Research reported in this publication was supported by the National Institute of General Medical Sciences of the National Institutes of Health under Award number R15GM099033.

\section{References}

1. Vasconcelos A, Cavaco-Paulo A. Curr. Drug Targets. 2013; 14:612. [PubMed: 23410124]

2. Cui L, Gong J, Fan X, Wang P, Wang Q, Qiu Y. Eng. Life Sci. 2013; 13(2):149.

3. Xu S, Sang L, Zhang Y, Wang X, Li X. Mater. Sci. Eng. C. 2013; 33:648.

4. de Guzman RC, Merrill MR, Richter JR, Hamzi RI, Greengauz Roberts OK, Dyke MEV. Biomaterials. 2011; 32:8205. [PubMed: 21835462]

5. Rouse JC, van Dyke ME. Materials. 2010; 3:999.

6. Tran CD, Duri S, Harkins AL. J. Biomed. Mat. Res. A. 2013; 101:2248.

7. Tran CD, Duri S, Delneri A, Franko M. J. Hazard. Mater. 2013; 252-253:355. [PubMed: 23542326]

8. Harkins AL, Duri S, Kloth LC, Tran CD. J. Biomed. Mat. Res. B. 2014; 00:000.

9. Xie H, Li S, Zhang S. Green Chem. 2005; 7:606.

10. Pinto RJB, Fernandes SCM, Freire CSR, Sadocco P, Causio J, Eto CP, Trindade T. Carbohydr. Res. 2012; 348:77. [PubMed: 22154478]

11. Altieri DC, Stamnes SJ, Gahmberg CG. Biochem. J. 1992; 288:465. [PubMed: 1361124]

12. Han KH, Chen Y, Chang MK, Han YC, Park JH, Green SR, Boullier A, Quehenberger O. J. Lipid Res. 2003; 44(7):1332. [PubMed: 12730301]

13. Tanabe T, Okitsu N, Tachibana A, Yamauchi K. Biomaterials. 2002; 23(3):817. [PubMed: 11771701] 


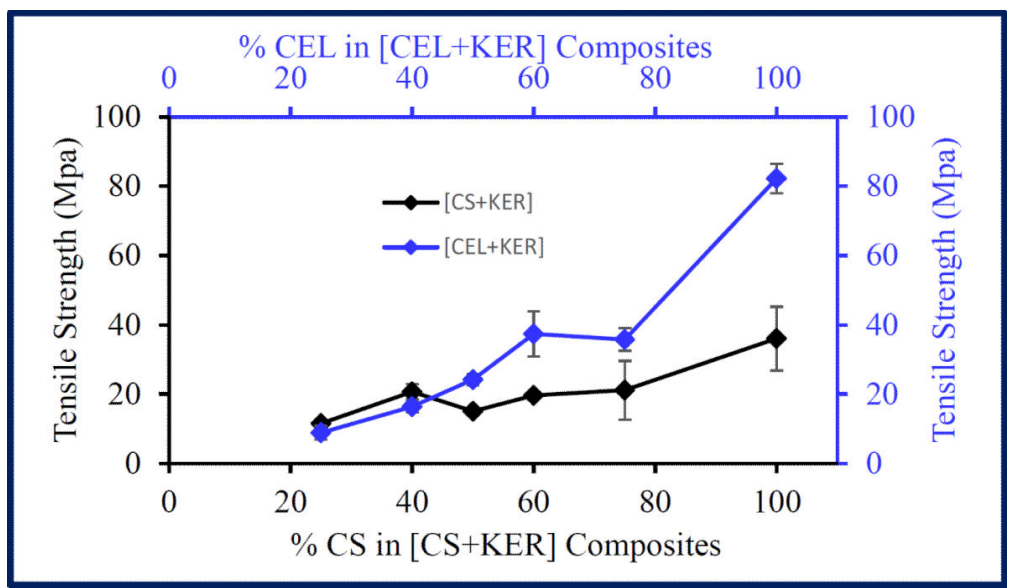

Figure 1.

Plot of tensile strength as a function of CEL and CS concentration, respectively in [KER $+\mathrm{CEL}]$ composites (blue curve) and [KER+CS] composite (black curve). 

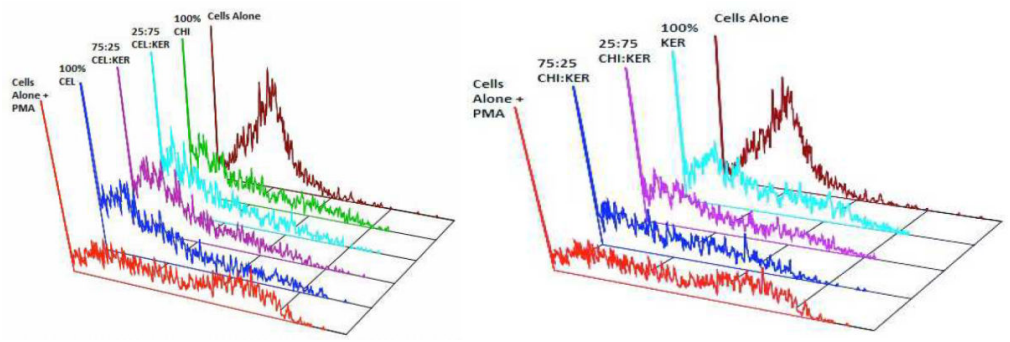

Figure 2.

Histograms displaying CD11b fluorescence from mitogen stimulated monocytes. PMAstimulated monocytes were incubated with (A) [CEL+KER] composites and 


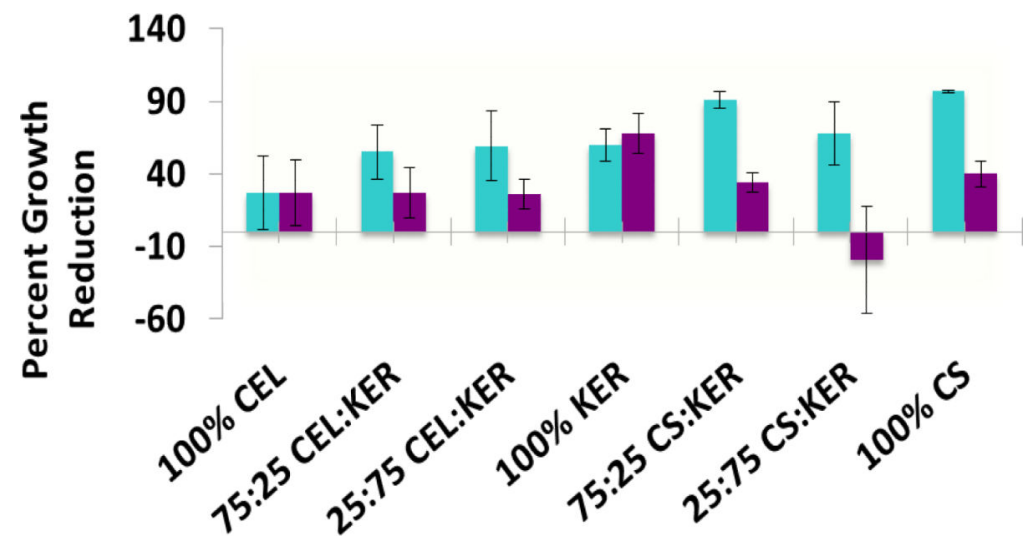

Figure 3.

Bacterial activity of composites. VRE (blue) and E. coli (purple) were cultured in the presence and absence of composites with different compositions and concentrations. The $\%$ growth reduction represents the $\mathrm{CFU} / \mathrm{mL}$ of bacteria divided by the $\mathrm{CFU} / \mathrm{ml}$ of the standard bacterial growth (i.e., no composite)X100. Each experiment was performed at least three independent times and the error bars represent standard deviation. 